\title{
Guaranteed recovery of unknown data from indirect noisy observations of their solutions on a finite system of points and intervals
}

\author{
Nakonechnyi O. G., Podlipenko Yu. K. \\ Taras Shevchenko National University of Kyiv, \\ 64/13 Volodymyrska Str., 01601, Kyiv, Ukraine
}

(Received 20 March 2019; Revised 22 April 2019; Accepted 25 April 2019)

\begin{abstract}
We consider the Cauchy problem for the first-order linear systems of ordinary differential equations with unknown right-hand sides and initial conditions that are supposed to be subjected to some quadratic restrictions. From indirect noisy observations of their solutions on a finite system of points and intervals, we obtain the linear guaranteed mean square estimates of linear functionals on unknown data of the above-mentioned problems. It is established that if the correlation functions of observational errors are not known and belong to special sets, such estimates are expressed via solutions to some boundary value problems for linear systems of impulsive ordinary differential equations.
\end{abstract}

Keywords: guaranteed mean square estimate, noisy observations, linear functionals from unknown data.

2000 MSC: $34 \mathrm{H} 05,34 \mathrm{G} 25$

UDC: 519.8

DOI: $10.23939 / \mathrm{mmc} 2019.02 .179$

\section{Introduction}

Estimation theory for systems with lumped and distributed parameters under uncertainty conditions was developed intensively during the last 30 years when essential results for ordinary and partial differential equations have been obtained. That was motivated by the fact that the realistic setting of boundary value problems describing physical processes often contains perturbations of unknown (or partially unknown) nature. In such cases, the minimax estimation method proved to be useful, making it possible to obtain optimal estimates both for the unknown solutions (or right-hand sides of equations appearing in the boundary value problems) and for linear functionals from them, that is, estimates looked for in the class of linear estimates with respect to observations ${ }^{1}$, for which the maximal mean square error taken over all the realizations of perturbations from the certain given sets takes its minimal value. Such estimates are called the guaranteed or minimax estimates.

Minimax estimation is studied in a big number of works; one may refer e.g. to [1-10] and the bibliography therein.

Let us formulate a general approach to the problem. If a state of a system is described by a linear ordinary differential equation

$$
\frac{d x(t)}{d t}=A x(t)+B v_{1}(t), \quad x\left(t_{0}\right)=x_{0},
$$

and a function $y(t)$ is observed in a time interval $\left[t_{0}, T\right]$, where $y(t)=H x(t)+v_{2}(t), x(t) \in \mathbb{R}^{n}$, $v_{2} \in \mathbb{R}^{m}, y \in \mathbb{R}^{m}$, and $A, B, H$ are known matrices, the minimax estimation problem consists in the most accurate determination of a function $x(t)$ at the "worst" realization of unknown quantities

\footnotetext{
${ }^{1}$ Here we understand observations of unknown solutions as the functions that are linear transformations of same solutions distorted by additive random noises.
} 
$\left(x_{0}, v_{1}(\cdot), v_{2}(\cdot)\right)$ taken from a certain set. N. N. Krasovskii was the first who stated this problem in [5]. Under different constraints imposed on function $v_{2}(t)$ and for known function $v_{1}(t)$ he proposed various methods of estimating inner products $(a, x(T))$ in the class of operations linear with respect to observations that minimize the maximal error. Later these estimates were called minimax a priori estimates (see $[3,5])$.

Fundamental results concerning estimation under uncertainties were obtained by A. B. Kurzhanskii $($ see $[3,4])$.

The duality principle elaborated in $[2,3,5]$ proved its efficiency for the determination of minimax estimates [2]. According to this principle, finding minimax a priori estimates can be reduced to a certain problem of optimal control of the system adjoint to (1); this approach enabled one to obtain, under certain restrictions, recurrent equations, namely, the minimax Kalman-Bucy filter (see [2]).

The present paper is devoted to the problems of guaranteed estimation for systems described by the Cauchy problem for first-order linear systems of ordinary differential differential equations with inexact data. From indirect noisy observations of unknown solutions on finite systems of points and intervals, under quadratic restrictions on unknown right-hand sides of equations and initial conditions, we find the guaranteed (minimax) estimates both for these right-hand sides and initial conditions and for linear functionals from them. It is proved that guaranteed estimates and estimation errors are expressed explicitly via the solutions of special boundary value problems for systems of linear impulsive ordinary differential equations, for which the unique solvability is established.

To do this, we reduce the guaranteed estimation problem to a certain optimal control problems. Solving this optimal control problems, we obtain the above mentioned boundary value problems that generate the minimax estimates.

\section{Problem statement}

Let vector-function $x(t) \in \mathbb{R}^{n}$ be a solution of the following Cauchy problem

$$
\begin{aligned}
\frac{d x(t)}{d t} & =A(t) x(t)+B(t) f(t), \quad t \in\left(t_{0}, T\right), \\
x\left(t_{0}\right) & =C x_{0},
\end{aligned}
$$

where $A(t)=\left[a_{i j}(t)\right]$ is an $n \times n$ matrix and $B(t)=\left[b_{i j}(t)\right]$ is an $n \times r$ matrix, whose entries $a_{i j}(t)$ and $b_{i j}(t)$ are continuous functions on the closed interval $\left[t_{0}, T\right], C=\left[c_{i j}\right]$ is an $n \times k$ real matrix, $f(t) \in \mathbb{R}^{r}$ is a vector-function belonging to the space $\left(L^{2}\left(t_{0}, T\right)\right)^{r}$, and $x_{0} \in \mathbb{R}^{k}$.

Here a solution $x(t)$ is interpreted as a continuous solution of the integral equation

$$
x(t)=x\left(t_{0}\right)+\int_{t_{0}}^{t}(A(s) x(s)+B(s) f(s)) d s
$$

or, equivalently, $x(t)$ satisfies (3), is absolutely continuous on $\left[t_{0}, T\right]$ with its derivative $x^{\prime}(t)$ satisfying (2) on $\left[t_{0}, T\right]$ almost everywhere (except on a set of Lebesgue measure 0 ), and belonging to $\left(L^{2}\left(t_{0}, T\right)\right)^{n}$.

Let $t_{1}, \ldots, t_{N}\left(t_{0}<t_{1}<\cdots<t_{N}<T\right)$ be a system of points on the closed interval $\left[t_{0}, T\right]$. Set $t_{N+1}=T$.

The problem is to determine a guaranteed mean square estimate of the value of the functional from $F=\left(x_{0}, f\right)$ of the form

$$
l(F)=\int_{t_{0}}^{T}\left(f(t), l_{0}(t)\right)_{r} d t+\left(x_{0}, a\right)_{k}
$$

from observations

$$
y_{i}=H_{i} x\left(t_{i}\right)+\xi_{i}, \quad i=1, \ldots, N,
$$




$$
y_{j}(t)=H_{j}(t) x(t)+\xi_{j}(t), \quad t \in \Omega_{j}, \quad j=1, \ldots, M
$$

in the class of estimates

$$
\widehat{l(F)}=\sum_{i=1}^{N}\left(y_{i}, u_{i}\right)_{m}+\sum_{j=1}^{M} \int_{\Omega_{j}}\left(y_{j}(t), u_{j}(t)\right)_{l} d t+c
$$

that are linear with respect to observations (5) and $(6)$. Here $(\cdot, \cdot)_{n}$ is the inner product in $\mathbb{R}^{n}, x(t)$ is the state of a system described by the Cauchy problem (2), (3), $l_{0} \in\left(L^{2}\left(t_{0}, T\right)\right)^{r}, a \in \mathbb{R}^{k}, H_{i}$ are $m \times n$ matrices, $H_{j}(t)$ are $l \times n$ matrices with the entries that are continuous functions on $\bar{\Omega}_{j}, u_{i} \in \mathbb{R}^{m}, u_{j}(t)$ are vector-functions belonging to $\left(L^{2}\left(\Omega_{j}\right)\right)^{l}$, and $c \in \mathbb{R}$. We suppose that $F:=\left(x_{0}, f\right) \in G_{1}$, where

$$
\begin{aligned}
G_{1}=\left\{\tilde{F}:=\left(\tilde{x}_{0}, \tilde{f}\right) \in \mathbb{R}^{k} \times\left(L^{2}\left(t_{0}, T\right)\right)^{r}:\right. & \left(Q_{0}\left(\tilde{x}_{0}-x_{0}^{0}\right), \tilde{x}_{0}-x_{0}^{0}\right)_{k} \\
& \left.+\int_{t_{0}}^{T}\left(Q_{1}(t)\left(\tilde{f}(t)-f_{0}(t)\right), \tilde{f}(t)-f_{0}(t)\right)_{r} d t \leqslant \varepsilon_{1}\right\},
\end{aligned}
$$

$\xi:=\left(\xi_{1}, \ldots, \xi_{N}, \xi_{1}(\cdot), \ldots, \xi_{M}(\cdot)\right) \in G_{2}, \xi_{i}=\left(\xi_{1}^{(i)}, \ldots, \xi_{m}^{(i)}\right)^{\mathrm{T}}$ and $\xi_{j}(\cdot)=\left(\xi_{1}^{(j)}(\cdot), \ldots, \xi_{l}^{(j)}(\cdot)\right)^{\mathrm{T}}$ are observation errors in (5) and (6), respectively, that are realizations of random vectors $\xi_{i}=\xi_{i}(\omega) \in \mathbb{R}^{m}$ and random vector-functions $\xi_{j}(t)=\xi_{j}(\omega, t) \in \mathbb{R}^{l}$ and $G_{2}$ denotes the set of random elements $\tilde{\xi}=$ $\left(\tilde{\xi}_{1}, \ldots, \tilde{\xi}_{N}, \tilde{\xi}_{1}(\cdot), \ldots, \tilde{\xi}_{M}(\cdot)\right)$, whose components $\tilde{\xi}_{i}=\left(\tilde{\xi}_{1}^{(i)}, \ldots, \tilde{\xi}_{m}^{(i)}\right)^{\mathrm{T}}$ and $\tilde{\xi}_{j}(\cdot)=\left(\tilde{\xi}_{1}^{(j)}(\cdot), \ldots, \tilde{\xi}_{l}^{(j)}(\cdot)\right)^{\mathrm{T}}$ are uncorrelated ${ }^{2}$, have zero means, $\mathbb{E} \tilde{\xi}_{i}=0$, and $\mathbb{E} \tilde{\xi}_{j}(\cdot)=0$, with finite second moments $\mathbb{E}\left|\tilde{\xi}_{i}\right|^{2}$ and $\mathbb{E}\left\|\tilde{\xi}_{j}(\cdot)\right\|_{\left(L^{2}\left(\Omega_{j}\right)\right)^{l}}^{2}$, and unknown correlation matrices $\tilde{R}_{i}=\mathbb{E} \tilde{\xi}_{i} \tilde{\xi}_{i}^{\mathrm{T}}=\left[r_{j k}^{(i)}\right]_{j, k=1}^{m}$ with entries $r_{j k}^{(i)}=\mathbb{E} \tilde{\xi}_{j}^{(i)} \tilde{\xi}_{k}^{(i)}$ and unknown correlation matrices $\tilde{R}_{j}(t, s)=\mathbb{E} \tilde{\xi}_{j}(t) \tilde{\xi}_{j}^{\mathrm{T}}(s)$ satisfying the conditions

$$
\sum_{i=1}^{N} \operatorname{Tr}\left[D_{i} \tilde{R}_{i}\right] \leqslant \varepsilon_{2}
$$

and

$$
\sum_{j=1}^{M} \int_{\Omega_{j}} \operatorname{Tr}\left[D_{j}(t) \tilde{R}_{j}(t, t)\right] d t \leqslant \varepsilon_{3},
$$

correspondingly, where $D_{i}=\left[d_{j k}^{(i)}\right]$ and $D_{j}(t)$ are symmetric positive definite $m \times m$ and $l \times l$ matrices, respectively $\left(\operatorname{Tr} D:=\sum_{i=1}^{l} d_{i i}\right.$ denotes the trace of the matrix $\left.D=\left\{d_{i j}\right\}_{i, j=1}^{l}\right)$. Here in $(7), x_{0}^{0} \in \mathbb{R}^{k}$, $f_{0} \in\left(L^{2}(0, T)\right)^{r}$ is a prescribed vector, $Q_{0}$ and $Q_{1}(t)$ are symmetric positive definite matrix and the entries of matices $D_{j}(t)$ and $Q_{1}(t)$ are assumed to be continuous on $\bar{\Omega}_{j}$ and $\left[t_{0}, T\right]$, respectively.

Set $u:=\left(u_{1}, \ldots, u_{N}, u_{1}(\cdot), \ldots, u_{M}(\cdot)\right) \in \mathbb{R}^{N \times m} \times\left(L^{2}\left(\Omega_{1}\right)\right)^{l} \times \cdots \times\left(L^{2}\left(\Omega_{M}\right)\right)^{l}=: H$. Norm in space $H$ is defined by

$$
\|u\|_{H}=\left\{\sum_{i=1}^{N}\left\|u_{i}\right\|_{\mathbb{R}^{m}}^{2}+\sum_{j=1}^{M}\left\|u_{j}(\cdot)\right\|_{\left(L^{2}\left(\Omega_{j}\right)\right)^{l}}^{2}\right\}^{1 / 2} .
$$

Definition 1. The guaranteed mean square estimate of expression (4) is the estimate

$$
\widehat{\widehat{l(F)}}=\sum_{i=1}^{N}\left(y_{i}, \hat{u}_{i}\right)_{m}+\sum_{j=1}^{M} \int_{\Omega_{j}}\left(y_{j}(t), \hat{u}_{j}(t)\right)_{l} d t+\hat{c},
$$

\footnotetext{
${ }^{2}$ That is, it is assumed that $\mathbb{E}\left(\tilde{\xi}_{i}, v\right)_{m}\left(\tilde{\xi}_{j}(\cdot), v(\cdot)\right)_{\left(L^{2}\left(\Omega_{j}\right)\right)^{l}}=0 \forall v \in \mathbb{R}^{m}, v(\cdot) \in\left(L^{2}\left(\Omega_{j}\right)\right)^{l}, i=1, \ldots N, j=1, \ldots M$.
} 
in which vectors $\hat{u}_{i}$, and a number $\hat{c}$ are determined from the condition

$$
\inf _{u \in H, c \in \mathbb{R}} \sigma(u, c)=\sigma(\hat{u}, \hat{c}),
$$

where

$$
\begin{gathered}
\sigma(u, c)=\sup _{\tilde{F} \in G_{1}, \tilde{\xi} \in G_{2}} \mathbb{E}|l(\tilde{F})-\widehat{l(\tilde{F})}|^{2}, \\
\widehat{l(\tilde{F})}=\sum_{i=1}^{N}\left(\tilde{y}_{i}, u_{i}\right)_{m}+\sum_{j=1}^{M} \int_{\Omega_{j}}\left(\tilde{y}_{j}(t), u_{j}(t)\right)_{l} d t+c, \\
\tilde{y}_{i}=H_{i} \tilde{x}\left(t_{i}\right)+\tilde{\xi}_{i}, \quad i=1, \ldots, N, \quad \tilde{y}_{j}(t)=H_{j}(t) \tilde{x}(t)+\tilde{\xi}_{j}(t), \quad j=1, \ldots, M,
\end{gathered}
$$

and $\tilde{x}(t)$ is the solution to the problem $(2),(3)$ at $f(t)=\tilde{f}(t), x_{0}=\tilde{x}_{0}$. The quantity

$$
\sigma:=\{\sigma(\hat{u}, \hat{c})\}^{1 / 2}
$$

is called the error of the guaranteed mean square estimation of $l(F)$.

Thus, a guaranteed mean square estimate is an estimate minimizing the maximal mean square estimation error calculated for the worst-case implementation of perturbations.

\section{Main results}

For any fixed $u:=\left(u_{1}, \ldots, u_{N}, u_{1}(\cdot), \ldots, u_{M}(\cdot)\right) \in H$ introduce vector-function $z(t ; u)$ as a unique solution to the problem ${ }^{3}$

$$
\begin{gathered}
-\frac{d z(t ; u)}{d t}=A^{\mathrm{T}}(t) z(t ; u)-\sum_{j=1}^{M} \chi_{\Omega_{j}}(t) H_{j}^{\mathrm{T}}(t) u_{j}(t), \quad t \in\left(t_{0}, T\right), \quad t \neq t_{i}, \\
\left.\Delta z(t ; u)\right|_{t=t_{i}}=z\left(t_{i}+0 ; u\right)-z\left(t_{i} ; u\right)=H_{i}^{\mathrm{T}} u_{i}, \quad i=1, \ldots, N, \quad z(T ; u)=0,
\end{gathered}
$$

where $\chi_{\Omega}(t)=\left\{\begin{array}{l}1 \text { if } t \in \Omega, \\ 0 \text { if } t \notin \Omega\end{array}\right.$ is the characteristic function of the set $\Omega, A^{\mathrm{T}}$ is the matrix transpose of $A$.

Lemma 1. Finding the guaranteed mean square estimate of functional $l(F)$ is equivalent to the problem of optimal control of the system (11)-(12) with the cost function

$$
\begin{aligned}
& I(u)=\varepsilon_{1}\left(\left(Q_{0}^{-1}\left(a+C^{\mathrm{T}} z\left(t_{0} ; u\right)\right), a+C^{\mathrm{T}} z\left(t_{0} ; u\right)\right)_{k}\right. \\
& \left.\quad+\int_{t_{0}}^{\mathrm{T}}\left(Q_{1}^{-1}(t)\left(l_{0}(t)+B^{\mathrm{T}}(t) z(t ; u)\right), l_{0}(t)+B^{\mathrm{T}}(t) z(t ; u)\right)_{r} d t\right) \\
& \quad+\varepsilon_{2} \sum_{i=1}^{N}\left(D_{i}^{-1} u_{i}, u_{i}\right)_{m}+\varepsilon_{3} \sum_{j=1}^{M} \int_{\Omega_{j}}\left(D_{j}^{-1}(t) u_{j}(t), u_{j}(t)\right)_{l} d t \rightarrow \inf _{u \in H} .
\end{aligned}
$$

Proof. For each $i=1, \ldots, N+1$, denote by $z_{i}(t ; u)$ the restriction of function $z(t ; u)$ to a subinterval $\left(t_{i-1}, t_{i}\right)$ of the interval $\left(t_{0}, T\right)$ and extend it from this subinterval to the ends $t_{i-1}$ and $t_{i}$ by continuity.

\footnotetext{
${ }^{3}$ Here and in what follows we assume that if a function is piecewise continuous then it is continuous from the left.
} 
Then

$$
\begin{aligned}
\frac{d z_{i}(t ; u)}{d t}+A^{\mathrm{T}}(t) z_{i}(t ; u) & =\sum_{j=1}^{M} \chi_{\Omega_{j}}(t) H_{j}^{\mathrm{T}}(t) u_{j}(t), \quad t_{i-1}<t<t_{i}, \quad i=1, \ldots, N+1, \\
z_{N+1}\left(t_{N+1} ; u\right) & =0, \quad z_{i+1}\left(t_{i} ; u\right)=z_{i}\left(t_{i} ; u\right)+H_{i}^{\mathrm{T}} u_{i}, \quad i=1, \ldots, N .
\end{aligned}
$$

Let $\tilde{x}$ be a solution to the problem $(2)$ and $(3)$ at $f(t)=\tilde{f}(t), x_{0}=\tilde{x}_{0}$. From relations (4) for $x=\tilde{x}$ and (10) and the integration by parts formula, we obtain

$$
\begin{gathered}
l(\tilde{F})-\widehat{l(\tilde{F})}=\int_{t_{0}}^{T}\left(\tilde{f}(t), l_{0}(t)\right)_{r} d t+\left(\tilde{x}_{0}, a\right)_{k}-\sum_{i=1}^{N}\left(\tilde{y}_{i}, u_{i}\right)_{m}-\sum_{j=1}^{M} \int_{\Omega_{j}}\left(\tilde{y}_{j}(t), u_{j}(t)\right)_{l} d t-c \\
=\int_{t_{0}}^{T}\left(\tilde{f}(t), l_{0}(t)\right)_{r} d t+\left(\tilde{x}_{0}, a\right)_{k}+\sum_{i=1}^{N+1} \int_{t_{i-1}}^{t_{i}}\left(\tilde{x}(t),-\frac{d z_{i}(t ; u)}{d t}-A^{\mathrm{T}}(t) z_{i}(t ; u)\right)_{n} d t-\sum_{i=1}^{N}\left(\tilde{x}\left(t_{i}\right), H_{i}^{\mathrm{T}} u_{i}\right)_{n} \\
-\sum_{i=1}^{N}\left(\tilde{\xi}_{i}, u_{i}\right)_{m}-\sum_{j=1}^{M} \int_{\Omega_{j}}\left(\tilde{\xi}_{j}(t), u_{j}(t)\right)_{l} d t-c \\
=\int_{t_{0}}^{T}\left(\tilde{f}(t), l_{0}(t)\right)_{r} d t+\left(\tilde{x}_{0}, a\right)_{k}+\sum_{i=1}^{N+1}\left(\left(\tilde{x}\left(t_{i-1}\right), z_{i}\left(t_{i-1} ; u\right)\right)_{n}-\left(\tilde{x}\left(t_{i}\right), z_{i}\left(t_{i} ; u\right)\right)_{n}\right) \\
+\sum_{i=1}^{N+1} \int_{t_{i-1}}^{t_{i}}\left(\frac{d \tilde{x}(t)}{d t}-A(t) \tilde{x}(t), z_{i}(t ; u)\right)_{n} d t \\
-\sum_{i=1}^{N}\left(\tilde{x}\left(t_{i}\right), z_{i+1}\left(t_{i} ; u\right)-z_{i}\left(t_{i} ; u\right)\right)_{n}-\sum_{i=1}^{N}\left(\tilde{\xi}_{i}, u_{i}\right)_{m}-\sum_{j=1}^{M} \int_{\Omega_{j}}\left(\tilde{\xi}_{j}(t), u_{j}(t)\right)_{l} d t-c \\
=\int_{t_{0}}^{T}\left(\tilde{f}(t), l_{0}(t)\right)_{r} d t+\left(\tilde{x}_{0}, a\right)_{k}+\left(\tilde{x}\left(t_{0}\right), z_{1}\left(t_{0} ; u\right)\right)_{n} \\
+\left(\tilde{x}\left(t_{1}\right), z_{1}\left(t_{1} ; u\right)\right)_{n}+\sum_{i=2}^{N}\left(\left(\tilde{x}\left(t_{i-1}\right), z_{i}\left(t_{i-1} ; u\right)\right)_{n}-\left(\tilde{x}\left(t_{i}\right), z_{i}\left(t_{i} ; u\right)\right)_{n}\right)+\left(\tilde{x}\left(t_{N}\right), z_{N+1}\left(t_{N}\right)\right)_{n} \\
+\sum_{i=1}^{N+1} \int_{t_{i-1}}^{t_{i}}\left(B(t) \tilde{f}(t), z_{i}(t ; u)\right)_{n} d t-\sum_{i=1}^{N}\left(\tilde{x}\left(t_{i}\right), z_{i+1}\left(t_{i} ; u\right)-z_{i}\left(t_{i} ; u\right)\right)_{n} \\
-\sum_{i=1}^{N}\left(\tilde{\xi}_{i}, u_{i}\right)_{m}-\sum_{j=1}^{M} \int_{\Omega_{j}}\left(\tilde{\xi}_{j}(t), u_{j}(t)\right)_{l} d t-c
\end{gathered}
$$

Taking into account that

$$
\begin{aligned}
\sum_{i=2}^{N}\left(\tilde{x}\left(t_{i-1}\right), z_{i}\left(t_{i-1} ; u\right)\right)_{n}+\left(\tilde{x}\left(t_{N}\right), z_{N+1}\left(t_{N}\right)\right)_{n} & =\sum_{i^{\prime}=1}^{N-1}\left(\tilde{x}\left(t_{i^{\prime}}\right), z_{i^{\prime}+1}\left(t_{i^{\prime}} ; u\right)\right)_{n}+\left(\tilde{x}\left(t_{N}\right), z_{N+1}\left(t_{N}\right)\right)_{n} \\
& =\sum_{i=1}^{N}\left(\tilde{x}\left(t_{i}\right), z_{i+1}\left(t_{i} ; u\right)\right)_{n},
\end{aligned}
$$

from latter equalities, we have

$$
l(\tilde{F})-\widehat{l(\tilde{F})}=\int_{t_{0}}^{T}\left(\tilde{f}(t), l_{0}(t)\right)_{r} d t+\left(\tilde{x}_{0}, a\right)_{k}+\left(\tilde{x}\left(t_{0}\right), z\left(t_{0} ; u\right)\right)_{n}
$$

Mathematical Modeling and Computing, Vol. 6, No. 2, pp. 179-191 (2019) 


$$
\begin{aligned}
&+\sum_{i=1}^{N+1} \int_{t_{i-1}}^{t_{i+1}}(\left.B(t) \tilde{f}(t), z_{i}(t ; u)\right)_{n} d t-\sum_{i=1}^{N}\left(\tilde{\xi}_{i}, u_{i}\right)_{m}-\sum_{j=1}^{M} \int_{\Omega_{j}}\left(\tilde{\xi}_{j}(t), \tilde{u}_{j}(t)\right)_{l} d t-c \\
&=\int_{t_{0}}^{T}\left(\tilde{f}(t), l_{0}(t)\right)_{r} d t+\left(\tilde{x}_{0}, a\right)_{k}+\left(C \tilde{x}_{0}, z\left(t_{0} ; u\right)\right)_{n} \\
&+\int_{t_{0}}^{T}(B(t) \tilde{f}(t), z(t ; u))_{n} d t-\sum_{i=1}^{N}\left(\tilde{\xi}_{i}, u_{i}\right)_{m}-\sum_{j=1}^{M} \int_{\Omega_{j}}\left(\tilde{\xi}_{j}(t), u_{j}(t)\right)_{l} d t-c .
\end{aligned}
$$

The latter relationship yields

$$
\mathbb{E}[l(\tilde{F})-\widehat{l(\tilde{F})}]=\left(\tilde{x}_{0}, a+C^{\mathrm{T}} z\left(t_{0} ; u\right)\right)_{k}+\int_{t_{0}}^{T}\left(\tilde{f}(t), l_{0}(t)+B^{\mathrm{T}}(t) z(t ; u)\right)_{r} d t-c .
$$

Taking into consideration the known relationship

$$
\mathbb{D} \eta=\mathbb{E}|\eta|^{2}-|\mathbb{E} \eta|^{2}
$$

that couples the variance $\mathbb{D} \eta=\mathbb{E}|\eta-\mathbb{E} \eta|^{2}$ of random variable $\eta$ with its expectation $\mathbb{E} \eta$, in which $\eta$ is determined by right-hand side of (14) and noncorrelatedness of $\tilde{\xi}_{i}=\left(\tilde{\xi}_{1}^{(i)}, \ldots, \tilde{\xi}_{m}^{(i)}\right)^{\mathrm{T}}$ and $\tilde{\xi}_{j}(\cdot)=$ $\left(\tilde{\xi}_{1}^{(j)}(\cdot), \ldots, \tilde{\xi}_{l}^{(j)}(\cdot)\right)^{\mathrm{T}}$, from the equalities (14) and (15) we find

$$
\begin{aligned}
\mathbb{E}|l(\tilde{F})-\widehat{l(\tilde{F})}|^{2}=\left|\left(\tilde{x}_{0}, a+C^{\mathrm{T}} z\left(t_{0} ; u\right)\right)_{k}+\int_{t_{0}}^{T}\left(\tilde{f}(t), l_{0}(t)+B^{\mathrm{T}}(t) z(t ; u)\right)_{r} d t-c\right|^{2} \\
\quad+\mathbb{E}\left|\sum_{i=1}^{N}\left(\tilde{\xi}_{i}, u_{i}\right)_{m}+\sum_{j=1}^{M} \int_{\Omega_{j}}\left(\tilde{\xi}_{j}(t), u_{j}(t)\right)_{l} d t\right|^{2} \\
=\mid\left(\tilde{x}_{0}-x_{0}^{0}, a+C^{\mathrm{T}} z\left(t_{0} ; u\right)\right)_{k}+\int_{t_{0}}^{T}\left(\tilde{f}(t)-f_{0}(t), l_{0}(t)+B^{\mathrm{T}}(t) z(t ; u)\right)_{r} d t \\
+\left(x_{0}^{0}, a+C^{\mathrm{T}} z\left(t_{0} ; u\right)\right)_{k}+\int_{t_{0}}^{T}\left(f_{0}(t), l_{0}(t)+B^{\mathrm{T}}(t) z(t ; u)\right)_{r} d t-\left.c\right|^{2} \\
\quad+\mathbb{E}\left|\sum_{i=1}^{N}\left(\tilde{\xi}_{i}, u_{i}\right)_{m}\right|^{2}+\mathbb{E}\left|\sum_{j=1}^{M} \int_{\Omega_{j}}\left(\tilde{\xi}_{j}(t), u_{j}(t)\right)_{l} d t\right|^{2} .
\end{aligned}
$$

Thus,

$$
\begin{aligned}
& \inf _{c \in \mathbb{R}} \sigma(u, c)=\inf _{c \in \mathbb{R}} \sup _{\tilde{F} \in G_{1}, \tilde{\xi} \in G_{2}} \mathbb{E}[l(\tilde{F})-\widehat{l(\tilde{F})}]^{2} \\
& =\inf _{c \in \mathbb{R}} \sup _{\tilde{F} \in G_{1}}\left[\left(\tilde{x}_{0}-x_{0}^{0}, a+C^{\mathrm{T}} z\left(t_{0} ; u\right)\right)_{k}+\int_{t_{0}}^{T}\left(\tilde{f}(t)-f_{0}(t), l_{0}(t)+B^{\mathrm{T}}(t) z(t ; u)\right)_{r} d t\right. \\
& \left.+\left(x_{0}^{0}, a+C^{\mathrm{T}} z\left(t_{0} ; u\right)\right)_{k}+\int_{t_{0}}^{T}\left(f_{0}(t), l_{0}(t)+B^{\mathrm{T}}(t) z(t ; u)\right)_{r} d t-c\right]^{2} \\
& \quad+\sup _{\tilde{\xi} \in G_{2}}\left(\mathbb{E}\left|\sum_{i=1}^{N}\left(\tilde{\xi}_{i}, u_{i}\right)_{m}\right|^{2}+\mathbb{E}\left|\sum_{j=1}^{M} \int_{\Omega_{j}}\left(\tilde{\xi}_{j}(t), u_{j}(t)\right)_{l} d t\right|^{2}\right) .
\end{aligned}
$$

Set

$$
y:=\left(\tilde{x}_{0}-x_{0}^{0}, a+C^{\mathrm{T}} z\left(t_{0} ; u\right)\right)_{k}+\int_{t_{0}}^{T}\left(\tilde{f}(t)-f_{0}(t), l_{0}(t)+B^{\mathrm{T}}(t) z(t ; u)\right)_{r} d t
$$




$$
d=c-\left(x_{0}^{0}, a+C^{\mathrm{T}} z\left(t_{0} ; u\right)\right)_{k}-\int_{t_{0}}^{T}\left(f_{0}(t), l_{0}(t)+B^{\mathrm{T}}(t) z(t ; u)\right)_{r} d t .
$$

Then for all $\tilde{F}=\left(\tilde{x}_{0}, \tilde{f}\right) \in G_{1}$, the generalized Cauchy-Bunyakovsky inequality and (7) imply

$$
\begin{aligned}
|y| \leqslant\left[\left(Q_{0}^{-1}(a+\right.\right. & \left.\left.C^{\mathrm{T}} z\left(t_{0} ; u\right)\right), a+C^{\mathrm{T}} z\left(t_{0} ; u\right)\right)_{k} \\
& \left.+\int_{t_{0}}^{T}\left(Q_{1}^{-1}(t)\left(l_{0}(t)+B^{\mathrm{T}}(t) z(t ; u)\right), l_{0}(t)+B^{\mathrm{T}}(t) z(t ; u)\right)_{r} d t\right]^{1 / 2} \\
\times & {\left[\left(Q_{0}\left(\tilde{x}_{0}-x_{0}^{0}\right), \tilde{x}_{0}-x_{0}^{0}\right)_{k}+\int_{t_{0}}^{T}\left(Q_{1}(t)\left(\tilde{f}(t)-f_{0}(t)\right), \tilde{f}(t)-f_{0}(t)\right)_{r} d t\right]^{1 / 2} \leqslant \varepsilon_{1}^{1 / 2} L }
\end{aligned}
$$

where

$$
\begin{aligned}
L=\left[\left(Q_{0}^{-1}\left(a+C^{\mathrm{T}} z\left(t_{0} ; u\right)\right), a+\right.\right. & \left.C^{\mathrm{T}} z\left(t_{0} ; u\right)\right)_{k} \\
& \left.+\int_{t_{0}}^{T}\left(Q_{1}^{-1}(t)\left(l_{0}(t)+B^{\mathrm{T}}(t) z(t ; u)\right), l_{0}(t)+B^{\mathrm{T}}(t) z(t ; u)\right)_{r} d t\right]^{1 / 2} .
\end{aligned}
$$

The direct substitution shows that the last inequality becomes the equality at $\tilde{F}=\left(\tilde{x}_{0}, \tilde{f}\right) \in G_{1}$, where

$$
\tilde{x}_{0}=x_{0}^{0} \pm \frac{\varepsilon_{1}^{1 / 2}}{L} Q_{0}^{-1}\left(a+C^{\mathrm{T}} z\left(t_{0} ; u\right)\right), \quad \tilde{f}(t)=f_{0}(t) \pm \frac{\varepsilon_{1}^{1 / 2}}{L} Q_{1}^{-1}(t)\left(l_{0}(t)+B^{\mathrm{T}}(t) z(t ; u)\right) .
$$

Taking into account

$$
\inf _{d \in \mathbb{R}} \sup _{|y| \leqslant \varepsilon_{1}^{1 / 2} L}|y-d|^{2}=\varepsilon_{1} L^{2}
$$

we find

$$
\begin{aligned}
& \inf _{c \in \mathbb{R}} \sup _{\tilde{F} \in G_{1}}\left[\left(a+C^{\mathrm{T}} z\left(t_{0} ; u\right), \tilde{x}_{0}-x_{0}^{0}\right)_{k}+\int_{t_{0}}^{T}\left(l_{0}(t)+B^{\mathrm{T}}(t) z(t ; u), \tilde{f}(t)-f_{0}(t)\right)\right)_{r} d t \\
&\left.+\left(a+C^{\mathrm{T}} z\left(t_{0} ; u\right), x_{0}^{0}\right)_{k}+\int_{t_{0}}^{T}\left(l_{0}(t)+B^{\mathrm{T}}(t) z(t ; u), f_{0}(t)\right)_{r} d t-c\right]^{2}=\varepsilon_{1} L^{2} \\
&=\varepsilon_{1}\left(Q_{0}^{-1}\left(a+C^{\mathrm{T}} z\left(t_{0} ; u\right)\right), a+C^{\mathrm{T}} z\left(t_{0} ; u\right)\right)_{k} \\
&+\varepsilon_{1} \int_{t_{0}}^{T}\left(Q_{1}^{-1}(t)\left(l_{0}(t)+B^{\mathrm{T}}(t) z(t ; u)\right), l_{0}(t)+B^{\mathrm{T}}(t) z(t ; u)\right)_{r} d t
\end{aligned}
$$

where the infimum over $c$ is attained at

$$
c=\left(a+C^{\mathrm{T}} z\left(t_{0} ; u\right), x_{0}^{0}\right)_{k}+\int_{t_{0}}^{T}\left(l_{0}(t)+B^{\mathrm{T}}(t) z(t ; u), f_{0}(t)\right)_{r} d t .
$$

Calculate the last term on the right-hand side of (16). Applying the generalized Cauchy-Bunyakovsky inequality, we have

$$
\mathbb{E}\left|\sum_{i=1}^{N}\left(\tilde{\xi}_{i}, u_{i}\right)_{m}\right|^{2} \leqslant \mathbb{E}\left[\sum_{i=1}^{N}\left(D_{i}^{-1} u_{i}, u_{i}\right)_{m} \cdot \sum_{i=1}^{N}\left(D_{i} \tilde{\xi}_{i}, \tilde{\xi}_{i}\right)_{m}\right]=\sum_{i=1}^{N}\left(D_{i}^{-1} u_{i}, u_{i}\right)_{m} \cdot \mathbb{E}\left[\sum_{i=1}^{N}\left(D_{i} \tilde{\xi}_{i}, \tilde{\xi}_{i}\right)_{m}\right] .
$$


Transform the last factor on the right-hand side of (19) as follows:

$$
\begin{aligned}
\mathbb{E}\left[\sum_{i=1}^{N}\left(D_{i} \tilde{\xi}_{i}, \tilde{\xi}_{i}\right)_{m}\right] & =\sum_{i=1}^{N} \mathbb{E}\left(\sum_{j=1}^{m} \sum_{k=1}^{m} d_{j k}^{(i)} \tilde{\xi}_{k}^{(i)} \tilde{\xi}_{j}^{(i)}\right) \\
& =\sum_{i=1}^{N} \sum_{j=1}^{m} \sum_{k=1}^{m} d_{j k}^{(i)} \mathbb{E} \tilde{\xi}_{k}^{(i)} \tilde{\xi}_{j}^{(i)}=\sum_{i=1}^{N} \sum_{j=1}^{m} \sum_{k=1}^{m} d_{j k}^{(i)} r_{k j}^{(i)}=\sum_{i=1}^{N} \operatorname{Tr}\left[D_{i} \tilde{R}_{i}\right]
\end{aligned}
$$

Analogously,

$$
\mathbb{E}\left|\sum_{j=1}^{M} \int_{\Omega_{j}}\left(\tilde{\xi}_{j}(t), u_{j}(t)\right)_{l} d t\right|^{2} \leqslant \sum_{j=1}^{M} \int_{\Omega_{j}}\left(D_{j}^{-1}(t) u_{j}(t), u_{j}(t)\right)_{l} d t \cdot \mathbb{E}\left[\sum_{j=1}^{M} \int_{\Omega_{j}}\left(D_{j}(t) \tilde{\xi}_{j}(t), \tilde{\xi}_{j}(t)\right)_{l} d t\right]
$$

and

$$
\mathbb{E}\left[\sum_{j=1}^{M} \int_{\Omega_{j}}\left(D_{j}(t) \tilde{\xi}_{j}(t), \tilde{\xi}_{j}(t)\right)_{l} d t\right]=\sum_{j=1}^{M} \int_{\Omega_{j}} \operatorname{Tr}\left[D_{j}(t) \tilde{R}_{j}(t, t)\right] d t
$$

Taking into account (8) and (9), we deduce from (19) that

$$
\begin{aligned}
\mathbb{E}\left|\sum_{i=1}^{N}\left(u_{i}, \tilde{\xi}_{i}\right)_{m}\right|^{2}+\mathbb{E}\left|\sum_{j=1}^{M} \int_{\Omega_{j}}\left(\tilde{\xi}_{j}(t), u_{j}(t)\right)_{l} d t\right|^{2} & \\
& \leqslant \varepsilon_{2} \sum_{i=1}^{N}\left(D_{i}^{-1} u_{i}, u_{i}\right)_{m}+\varepsilon_{3} \sum_{j=1}^{M} \int_{\Omega_{j}}\left(D_{j}^{-1}(t) u_{j}(t), u_{j}(t)\right)_{l} d t
\end{aligned}
$$

It is not difficult to check that the equality sign is attained at the element

$$
\xi^{(0)}=\left(\xi_{1}^{(0)}, \ldots, \xi_{N}^{(0)}, \xi_{1}^{(0)}(\cdot), \ldots, \xi_{M}^{(0)}(\cdot)\right) \in G_{2}
$$

with

$$
\begin{gathered}
\xi_{i}^{(0)}=\frac{\varepsilon_{2}^{1 / 2} \eta_{1} D_{i}^{-1} u_{i}}{\left[\sum_{i=1}^{N}\left(D_{i}^{-1} u_{i}, u_{i}\right)_{m}\right]^{1 / 2}}, \quad i=1, \ldots, N, \\
\xi_{j}^{(0)}(t)=\frac{\varepsilon_{3}^{1 / 2} \eta_{2} D_{j}^{-1}(t) u_{j}(t)}{\left[\sum_{j=1}^{M} \int_{\Omega_{j}}\left(D_{j}^{-1}(t) u_{j}(t), u_{j}(t)\right)_{l} d t\right]^{1 / 2}}, \quad j=1, \ldots, M,
\end{gathered}
$$

where $\eta_{1}$ and $\eta_{2}$ are uncorrelated random variables such that $\mathbb{E} \eta_{i}=0$ and $\mathbb{E}\left|\eta_{i}\right|^{2}=1, i=1,2$. Hence,

$$
\begin{aligned}
\sup _{\tilde{\xi} \in G_{2}}\left(\mathbb{E}\left|\sum_{i=1}^{N}\left(\tilde{\xi}_{i}, u_{i}\right)_{m}\right|^{2}+\mathbb{E} \mid \sum_{j=1}^{M} \int_{\Omega_{j}}\right. & \left.\left.\left(\tilde{\xi}_{j}(t), u_{j}(t)\right)_{l} d t\right|^{2}\right) \\
& =\varepsilon_{2} \sum_{i=1}^{N}\left(D_{i}^{-1} u_{i}, u_{i}\right)_{m}+\varepsilon_{3} \sum_{j=1}^{M} \int_{\Omega_{j}}\left(D_{j}^{-1}(t) u_{j}(t), u_{j}(t)\right)_{l} d t
\end{aligned}
$$

The statement of the lemma follows now from (16), (17), (18), and (20). The proof is complete.

Using this lemma, we obtain the following result.

Mathematical Modeling and Computing, Vol. 6, No. 2, pp. 179-191 (2019) 
Theorem 2. The guaranteed mean square estimate $\widehat{\overline{l(F)}}$ of expression $l(F)$ has the form

$$
\widehat{\widehat{l(F)}}=\sum_{i=1}^{N}\left(y_{i}, \hat{u}_{i}\right)_{m}+\sum_{j=1}^{M} \int_{\Omega_{j}}\left(y_{j}(t), \hat{u}_{j}(t)\right)_{l} d t+\hat{c}=l(\hat{F}),
$$

where

$$
\begin{gathered}
\hat{u}_{i}=\varepsilon_{2}^{-1} D_{i} H_{i} p\left(t_{i}\right), \quad i=1, \ldots, N, \quad \hat{u}_{j}(t)=\varepsilon_{3}^{-1} D_{j}(t) H_{j}(t) p(t), \quad j=1, \ldots, M, \\
\hat{c}=\left(x_{0}^{0}, a+C^{\mathrm{T}} \hat{z}\left(t_{0} ; u\right)\right)_{k}+\int_{t_{0}}^{T}\left(f_{0}(t), l_{0}(t)+B^{\mathrm{T}}(t) \hat{z}(t)\right)_{r} d t \\
\hat{F}=\left(\hat{x}_{0}, \hat{f}\right) \quad \text { with } \quad \hat{x}_{0}=x_{0}^{0}+\varepsilon_{1} Q_{0}^{-1}(t) C^{\mathrm{T}} \hat{p}\left(t_{0}\right), \quad \hat{f}(t)=f_{0}(t)+\varepsilon_{1} Q_{1}^{-1}(t) B^{\mathrm{T}}(t) \hat{p}(t),
\end{gathered}
$$

and the vector-functions $p(t), \hat{z}(t)$, and $\hat{p}(t)$ are determined from the solutions of the problems

$$
\begin{gathered}
-\frac{d \hat{z}(t)}{d t}=A^{\mathrm{T}}(t) \hat{z}(t)-\varepsilon_{3}^{-1} \sum_{j=1}^{M} \chi_{\Omega_{j}}(t) H_{j}^{\mathrm{T}}(t) D_{j}(t) H_{j}(t) p(t), \quad t \in\left(t_{0}, T\right), \quad t \neq t_{i}, \\
\left.\Delta \hat{z}(t)\right|_{t=t_{i}}=\hat{z}\left(t_{i}+0\right)-\hat{z}\left(t_{i}\right)=\varepsilon_{2}^{-1} H_{i}^{\mathrm{T}} D_{i} H_{i} p\left(t_{i}\right), \quad i=1, \ldots, N, \quad \hat{z}(T)=0, \\
\frac{d p(t)}{d t}=A(t) p(t)+\varepsilon_{1} B(t) Q_{1}^{-1}(t)\left(B^{\mathrm{T}} \hat{z}(t)+l_{0}(t)\right), \quad t \in\left(t_{0}, T\right), \quad t \neq t_{i}, \\
\left.\Delta p(t)\right|_{t=t_{i}}=p\left(t_{i}+0\right)-p\left(t_{i}\right)=0, \quad i=1, \ldots, N, \quad p\left(t_{0}\right)=\varepsilon_{1} C Q_{0}^{-1}\left(C^{\mathrm{T}} \hat{z}\left(t_{0}\right)+a\right)
\end{gathered}
$$

and

$$
\begin{gathered}
-\frac{d \hat{p}(t)}{d t}=A^{\mathrm{T}}(t) \hat{p}(t)-\varepsilon_{3}^{-1} \sum_{j=1}^{M} \chi_{\Omega_{j}}(t) H_{j}^{\mathrm{T}}(t) D_{j}(t)\left[H_{j}(t) \hat{x}(t)-y_{j}(t)\right], \quad t \in\left(t_{0}, T\right), \quad t \neq t_{i}, \\
\left.\Delta \hat{p}(t)\right|_{t=t_{i}}=\hat{p}\left(t_{i}+0\right)-\hat{p}\left(t_{i}\right)=\varepsilon_{2}^{-1} H_{i}^{\mathrm{T}} D_{i}\left[H_{i} \hat{x}\left(t_{i}\right)-y_{i}\right], \quad i=1, \ldots, N, \quad \hat{p}(T)=0, \\
\frac{d \hat{x}(t)}{d t}=A(t) \hat{x}(t)+\varepsilon_{1} \tilde{Q}_{1}(t) \hat{p}(t)+B(t) f_{0}(t), \quad t \in\left(t_{0}, T\right), \quad t \neq t_{i}, \\
\left.\Delta \hat{x}(t)\right|_{t=t_{i}}=\hat{x}\left(t_{i}+0\right)-\hat{x}\left(t_{i}\right)=0, \quad i=1, \ldots, N, \quad \hat{x}\left(t_{0}\right)=\varepsilon_{1} \tilde{Q}_{0} \hat{p}\left(t_{0}\right)+C x_{0}^{0} .
\end{gathered}
$$

respectively. Here, $\tilde{Q}_{0}=C Q_{0}^{-1} C^{\mathrm{T}}, \tilde{Q}_{1}(t)=B(t) Q_{1}^{-1}(t) B^{T}(t)$. Problems $(21)-(24)$ and $(25)-(28)$ are uniquely solvable. Equations $(25)-(28)$ are fulfilled with probability 1.

The guaranteed mean square estimation error is determined by the formula

$$
\sigma=[l(\hat{P})]^{1 / 2},
$$

where

$$
\hat{P}=\left(\varepsilon_{1} Q_{0}^{-1}\left(a+C^{T} \hat{z}\left(t_{0}\right)\right), \varepsilon_{1} Q_{1}^{-1}(t)\left(l_{0}(t)+B^{T}(t) \hat{z}(t)\right)\right)
$$

Proof. From the results contained in [11, Chapter 1], it follows the estimates

$$
\left\|z\left(t_{0} ; u\right)\right\|_{n} \leqslant c_{1}\|u\|_{H}, \quad\|z(\cdot ; u)\|_{\left(L^{2}\left(t_{0}, T\right)\right)^{n}} \leqslant c_{2}\|u\|_{H},
$$

where $c_{1}, c_{2} \in \mathbb{R}$. Taking into account these inequalities, one can easily verify that $I(u)$ is a continuous strictly convex functional on $H$. By [12, Corollary 1.8.3], $I(u)$ is a weak lower semicontinuous strictly 
convex functional on $H$. Since

$$
I(u) \geqslant \varepsilon_{2} \sum_{i=1}^{N}\left(D_{i}^{-1} u_{i}, u_{i}\right)_{m}+\varepsilon_{3} \sum_{j=1}^{M} \int_{\Omega_{j}}\left(D_{j}^{-1}(t) u_{j}(t), u_{j}(t)\right)_{l} d t \geqslant c\|u\|_{H}^{2} \quad \forall u \in H, \quad c \in \mathbb{R},
$$

by [13, Theorems 13.2 and 13.4] there exists one and only one element $\hat{u} \in H$ such that $I(\hat{u})=\inf _{u \in H} I(u)$. Hence, for any fixed $v \in H$ and $\tau \in \mathbb{R}$ the function $s(\tau):=I(\hat{u}+\tau v)$ reaches its minimum at a unique point $\tau=0$, so that

$$
\left.\frac{1}{2} \frac{d}{d \tau} I(\hat{u}+\tau v)\right|_{\tau=0}=0
$$

Since $z(t ; \hat{u}+\tau v)=z(t ; \hat{u})+\tau z(t ; v)$, we obtain from (13) and (30) that

$$
\begin{aligned}
& 0=\varepsilon_{1} \sum_{i=1}^{N+1} \int_{t_{i-1}}^{t_{i}}\left(Q _ { 1 } ^ { - 1 } ( t ) \left(B^{\mathrm{T}}(t) z_{i}(t\right.\right.\left.\left.; \hat{u})+l_{0}(t)\right), B^{\mathrm{T}}(t) z_{i}(t ; v)\right)_{r} d t \\
&+\varepsilon_{1}\left(Q_{0}^{-1}\left(C^{\mathrm{T}} z_{1}\left(t_{0} ; \hat{u}\right)+a\right), C^{\mathrm{T}} z_{1}\left(t_{0} ; v\right)\right)_{k} \\
& \quad+\varepsilon_{2} \sum_{i=1}^{N}\left(D_{i}^{-1} \hat{u}_{i}, v_{i}\right)_{m}+\varepsilon_{3} \sum_{j=1}^{M} \int_{\Omega_{j}}\left(D_{j}^{-1}(t) \hat{u}_{j}(t), v_{j}(t)\right)_{l} d t
\end{aligned}
$$

Let $p(t)$ be a solution of the problem

$$
\begin{gathered}
\frac{d p(t)}{d t}=A(t) p(t)+\varepsilon_{1} B(t) Q_{1}^{-1}(t)\left(B^{\mathrm{T}} z(t ; \hat{u})+l_{0}(t)\right), \quad t \in\left(t_{0}, T\right), \quad t \neq t_{i}, \\
\left.\Delta p(t)\right|_{t=t_{i}}=p\left(t_{i}+0\right)-p\left(t_{i}\right)=0, \quad i=1, \ldots, N, \quad p\left(t_{0}\right)=\varepsilon_{1} C Q_{0}^{-1}\left(C^{\mathrm{T}} z\left(t_{0} ; \hat{u}\right)+a\right) .
\end{gathered}
$$

For each $i=1, \ldots, N+1$, let $p_{i}(t)$ be the restriction of function $p(t)$ to a subinterval $\left(t_{i-1}, t_{i}\right)$ of the interval $\left(t_{0}, T\right)$ that is extended from this subinterval to its ends $t_{i-1}$ and $t_{i}$ by continuity. Then

$$
\begin{gathered}
\frac{d p_{i}(t)}{d t}-A(t) p_{i}(t)=\varepsilon_{1} B(t) Q_{1}^{-1}(t)\left(B^{\mathrm{T}} z_{i}(t ; \hat{u})+l_{0}(t)\right), \quad t_{i-1}<t<t_{i}, \quad i=1, \ldots, N+1, \\
p_{1}\left(t_{0}\right)=\varepsilon_{1} C Q_{0}^{-1}\left(C^{\mathrm{T}} z_{1}\left(t_{0} ; \hat{u}\right)+a\right), \quad p_{i+1}\left(t_{i}\right)=p_{i}\left(t_{i}\right), \quad i=1, \ldots, N .
\end{gathered}
$$

Taking into account $z_{N+1}\left(t_{N+1} ; v\right)=z_{N+1}(T ; v)=0,(23)$, and (24), we have

$$
\begin{gathered}
\varepsilon_{1} \sum_{i=1}^{N+1} \int_{t_{i-1}}^{t_{i}}\left(Q_{1}^{-1}(t)\left(B^{\mathrm{T}}(t) z_{i}(t ; \hat{u})+l_{0}(t)\right), B^{\mathrm{T}}(t) z_{i}(t ; v)\right)_{r} d t+\varepsilon_{1}\left(Q_{0}^{-1}\left(C^{\mathrm{T}} z_{1}\left(t_{0} ; \hat{u}\right)+a\right), C^{\mathrm{T}} z_{1}\left(t_{0} ; v\right)\right)_{k} \\
=\sum_{i=1}^{N+1} \int_{t_{i-1}}^{t_{i}}\left(\frac{d p_{i}(t)}{d t}-A(t) p_{i}(t), z_{i}(t ; v)\right)_{n} d t+\left(p_{1}\left(t_{0}\right), z_{1}\left(t_{0} ; v\right)\right)_{n} \\
=-\left(p_{N+1}\left(t_{N}\right), z_{N+1}\left(t_{N} ; v\right)\right)_{n}+\sum_{i=1}^{N}\left(\left(p_{i}\left(t_{i}\right), z_{i}\left(t_{i} ; v\right)\right)_{n}-\left(p_{i}\left(t_{i-1}\right), z_{i}\left(t_{i-1} ; v\right)\right)_{n}\right)+\left(p_{1}\left(t_{0}\right), z_{1}\left(t_{0} ; v\right)\right)_{n} \\
+\sum_{i=1}^{N+1} \int_{t_{i-1}}^{t_{i}}\left(-\frac{d z_{i}(t ; v)}{d t}-A^{\mathrm{T}}(t) z_{i}(t ; v), p_{i}(t ; v)\right)_{n} d t \\
=\sum_{i=1}^{N}\left(p_{i}\left(t_{i}\right), z_{i}\left(t_{i} ; v\right)\right)_{n}-\sum_{i=2}^{N+1}\left(p_{i}\left(t_{i-1}\right), z_{i}\left(t_{i-1} ; v\right)\right)_{n}-\sum_{j=1}^{M} \int_{\Omega_{j}}\left(p(t), H_{j}^{\mathrm{T}}(t) v_{j}(t)\right)_{n} d t \\
=\left(p_{1}\left(t_{1}\right), z_{1}\left(t_{1} ; v\right)\right)_{n}-\left(p_{2}\left(t_{1}\right), z_{2}\left(t_{1} ; v\right)\right)_{n}+\cdots+\left(p_{i}\left(t_{i}\right), z_{i}\left(t_{i} ; v\right)\right)_{n}-\left(p_{i+1}\left(t_{i}\right), z_{i+1}\left(t_{i} ; v\right)\right)_{n}
\end{gathered}
$$

Mathematical Modeling and Computing, Vol.6, No. 2, pp. 179-191 (2019) 


$$
\begin{gathered}
+\cdots+\left(p_{N}\left(t_{N}\right), z_{N}\left(t_{N} ; v\right)\right)_{n}-\left(p_{N+1}\left(t_{N}\right), z_{N+1}\left(t_{N} ; v\right)\right)_{n}-\sum_{j=1}^{M} \int_{\Omega_{j}}\left(p(t), H_{j}^{\mathrm{T}}(t) v_{j}(t)\right)_{n} d t \\
\left.=\left(p_{1}\left(t_{1}\right), z_{1}\left(t_{1} ; v\right)-z_{2}\left(t_{1} ; v\right)\right)_{n}+\cdots+\left(p_{i}\left(t_{i}\right), z_{i}\left(t_{i} ; v\right)\right)_{n}-z_{i+1}\left(t_{i} ; v\right)\right)_{n} \\
\left.+\cdots+\left(p_{N}\left(t_{N}\right), z_{N}\left(t_{N} ; v\right)\right)_{n}-z_{N+1}\left(t_{N} ; v\right)\right)_{n}=-\sum_{i=1}^{N}\left(p_{i}\left(t_{i}\right), H_{i}^{\mathrm{T}} v_{i}\right)_{n}-\sum_{j=1}^{M} \int_{\Omega_{j}}\left(p(t), H_{j}^{\mathrm{T}}(t) v_{j}(t)\right)_{n} d t .
\end{gathered}
$$

From (31) and (32), we find

$$
\hat{u}_{i}=\varepsilon_{2}^{-1} D_{i} H_{i} p_{i}\left(t_{i}\right), \quad i=1, \ldots, N, \quad \hat{u}_{j}(t)=\varepsilon_{3}^{-1} D_{j}(t) H_{j}(t) p(t), \quad j=1, \ldots, M .
$$

Setting

$$
\begin{aligned}
u=\hat{u}=\left(\varepsilon_{2}^{-1} D_{1} H_{1} p\left(t_{1}\right), \ldots,\right. & \varepsilon_{2}^{-1} D_{i} H_{i} p\left(t_{i}\right), \ldots, \varepsilon_{2}^{-1} D_{N} H_{N} p\left(t_{N}\right), \\
& \left.\varepsilon_{3}^{-1} D_{1}(t) H_{1}(t) p(t), \ldots, \varepsilon_{3}^{-1} D_{j}(t) H_{j}(t) p(t), \ldots, \varepsilon_{3}^{-1} D_{M}(t) H_{M}(t) p(t)\right)
\end{aligned}
$$

in (11), (12), and (18), and denoting $\hat{z}(t)=z(t ; \hat{u})$, we see that $\hat{z}(t)$ and $p(t)$ satisfy system $(21)-(24)$; the unique solvability of this system follows from the fact that the functional $I(u)$ has one minimum point $\hat{u}$.

Let us prove that $\sigma=[l(\hat{P})]^{1 / 2}$. Substituting expression (33) to (13), we obtain

$$
\begin{aligned}
\sigma^{2}=\sigma(\hat{u}, \hat{c})= & \varepsilon_{1} \sum_{i=1}^{N+1} \int_{t_{i-1}}^{t_{i}}\left(Q_{1}^{-1}(t)\left(l_{0}(t)+B^{\mathrm{T}}(t) \hat{z}_{i}(t)\right), l_{0}(t)+B^{\mathrm{T}}(t) \hat{z}_{i}(t)\right)_{r} d t \\
& \quad+\varepsilon_{1}\left(Q_{0}^{-1}\left(a+C^{\mathrm{T}} \hat{z}\left(t_{0}\right)\right), a+C^{\mathrm{T}} \hat{z}\left(t_{0}\right)\right)_{k} \\
& +\varepsilon_{2}^{-1} \sum_{i=1}^{N}\left(H_{i} p_{i}\left(t_{i}\right), D_{i} H_{i} p_{i}\left(t_{i}\right)\right)_{m}+\varepsilon_{3}^{-1} \sum_{j=1}^{M} \int_{\Omega_{j}}\left(H_{j}(t) p(t), D_{j}(t) H_{j}(t) p(t)\right)_{l} d t
\end{aligned}
$$

However,

$$
\begin{gathered}
\varepsilon_{1} \sum_{i=1}^{N+1} \int_{t_{i-1}}^{t_{i}}\left(Q_{1}^{-1}(t)\left(l_{0}(t)+B^{\mathrm{T}}(t) \hat{z}_{i}(t)\right), l_{0}(t)+B^{\mathrm{T}}(t) \hat{z}_{i}(t)\right)_{r} d t+\varepsilon_{1}\left(Q_{0}^{-1}\left(a+C^{\mathrm{T}} \hat{z}\left(t_{0}\right)\right), a+C^{\mathrm{T}} \hat{z}\left(t_{0}\right)\right)_{k} \\
=\sum_{i=1}^{N+1} \int_{t_{i-1}}^{t_{i}}\left(\frac{d p_{i}(t)}{d t}-A(t) p_{i}(t), \hat{z}_{i}(t)\right)_{n} d t+\varepsilon_{1} \int_{t_{0}}^{T}\left(Q_{1}^{-1}(t)\left(l_{0}(t)+B^{\mathrm{T}}(t) \hat{z}(t)\right), l_{0}(t)\right)_{r} d t \\
+\left(p_{1}\left(t_{0}\right), \hat{z}_{1}\left(t_{0}\right)\right)_{n}+\varepsilon_{1}\left(Q_{0}^{-1}\left(a+C^{\mathrm{T}} \hat{z}\left(t_{0}\right)\right), a\right)_{k}=l(\hat{P})+\sum_{i=1}^{N+1}\left(\left(p_{i}\left(t_{i}\right), \hat{z}_{i}\left(t_{i}\right)\right)_{n}-\left(p_{i}\left(t_{i-1}\right), \hat{z}_{i}\left(t_{i-1}\right)\right)_{n}\right) \\
+\sum_{i=1}^{N+1} \int_{t_{i-1}}^{t_{i}}\left(-\frac{d \hat{z}_{i}(t)}{d t}-A^{\mathrm{T}}(t) \hat{z}_{i}(t), p_{i}(t)\right)_{n} d t+\left(p_{1}\left(t_{0}\right), \hat{z}_{1}\left(t_{0}\right)\right)_{n} \\
=l(\hat{P})-\varepsilon_{3}^{-1} \sum_{j=1}^{M} \int_{\Omega_{j}}\left(p(t), H_{j}^{\mathrm{T}}(t) D_{j}(t) H_{j}(t) p(t)\right)_{n} d t-\varepsilon_{2}^{-1} \sum_{i=1}^{N}\left(p_{i}\left(t_{i}\right), H_{i}^{\mathrm{T}} D_{i} H_{i} p_{i}\left(t_{i}\right)\right)_{n} . \quad(35)
\end{gathered}
$$

Then (29) follows from (34) and (35). 
The representation

$$
\widehat{\widehat{l(F)}}=l(\hat{F}) .
$$

can be proved in much the same way as the representation

$$
\widehat{\widehat{l(F)}}=\sum_{i=1}^{N}\left(y_{i}, \hat{u}_{i}\right)_{m}+\sum_{j=1}^{M} \int_{\Omega_{j}}\left(y_{j}(t), \hat{u}_{j}(t)\right)_{l} d t+\hat{c} .
$$

This completes the proof.

Remark 1. In the representation $\widehat{\widehat{l(F)}}=l(\hat{F})$ of the guaranteed mean square estimate of $l(F)$, where $\hat{F}=\left(\hat{x}_{0}, \hat{f}\right)$ and $F=\left(x_{0}, f\right)$, the vector $\hat{x}_{0}=x_{0}^{0}+\varepsilon_{1} Q_{0}^{-1}(t) C^{\mathrm{T}} \hat{p}\left(t_{0}\right)$ and the vector-function $\hat{f}(t)=f_{0}(t)+\varepsilon_{1} Q_{1}^{-1}(t) B^{\mathrm{T}}(t) \hat{p}(t)$ do not depend on a specific form of functional $l$. Therefore, $\hat{x}_{0}$ and $\hat{f}(t)$ can be taken as good estimates of unknown $x_{0}$ and $f(t)$, respectively.

Remark 2. When observations are pointwise (i.e., $H_{j}(t)=0$ and $\xi_{j}(t)=0, j=1, \ldots, M$ in (6)), the systems of ODEs (21)-(24) and (25)-(28) are equivalent to some systems of linear algebraic equations [14].

\section{Conclusion}

We elaborate a minimax approach to the problem of estimation of unknown data for systems governed by the Cauchy problem for first-order linear systems of ordinary differential equations from noisy observations of their solutions. Here we use a new class of observations distributed on a finite system of points and intervals.

It has been established that the guaranteed mean square estimates are expressed via solutions of some linear systems of impulsive ordinary differential equations.

The obtained systems of ordinary differential equations that generate the guaranteed mean square estimates of linear functionals can be applied to the processing of information for estimation of mean values of stochastic vector processes from their observations distorted by noises, whose correlation functions are unknown.

[1] Nakonechnyi O. G. Minimax Estimates in Systems with Distributed Parameters. Preprint 79 Acad. Sci. USSR, Inst. Cybernetics, Kyiv (1979).

[2] Kirichenko N. F., Nakonechnyi O. G. Minimax approach to recursive estimation of states of linear dynamic systems. Cybernetics. 13 (4), 527-531 (1977).

[3] Kurzhanskii A. B. Control and Observation under Uncertainties. Nauka, Moscow (1977), (in Russian).

[4] Kurzhanski A. B., Valyi I. Ellipsoidal Calculus for Estimation and Control. Birkhäuser, Boston (1997).

[5] Krasovskii N. N. Theory of Motion Control. Nauka, Moscow (1968), (in Russian).

[6] Nakonechnyi O. G. Optimal Control and Estimation for Partial Differential Equations. Kyiv University, Kyiv (2004).

[7] Podlipenko Yu., Shestopalov Yu. Guaranteed estimation of solutions to Helmholtz transmission problems with uncertain data from their indirect noisy observations. Radio Science. 52 (9), 1129-1139 (2017).

[8] Podlipenko Yu., Shestopalov Yu. Mixed variational approach to finding guaranteed estimates from solutions and right-hand sides of the second-order linear elliptic equations under incomplete data. Minimax Theory and its Applications. 1 (2), 197-244, (2016).

[9] Shestopalov Y., Podlipenko Y., Nakonechnyi O. Estimation of Parameters of Boundary Value Problems for Linear Ordinary Differential Equations with Uncertain Data. Advances in Pure Mathematics. 4 (4), $118-146$ (2014). 
[10] Zhuk S., Nakonechnii O. Minimax State Estimates for Abstract Neumann Problems. Minimax Theory and its Applications. 3 (1), 1-21 (2018).

[11] Bainov D. D., Simeonov P.S. Impulsive Differential Equations: Asymptotic Properties of the Solutions. World Scientifc (1995).

[12] Balakrishnan A. V. Applied Functional Analysis. Springer-Verlag; 2nd edition (1981).

[13] Badriev I. B., Karchevsky M. M. Duality methods in applied problems. Publ. of Kazan State University, Kazan (1987).

[14] Nakonechny O. G., Podlipenko Yu. K. The minimax approach to the estimation of solutions to first order linear systems of ordinary differential periodic equations with inexact data. arXiv:1810.07228V1, p.19 (2018).

\title{
Гарантоване відновлення невідомих даних за непрямими зашумленими спостереженнями їх розв'язків на скінченній системі точок і інтервалів
}

\author{
Наконечний О. Г., Подлипенко Ю. К. \\ Київсъкий національний університет імені Тараса Шевченка, \\ вул. Володимирсъка, 64/13, Київ, 01601, Україна
}

\begin{abstract}
Розглянуто задачу Коші для лінійних систем звичайних диференціальних рівнянь першого порядку з невідомими правими частинами і початковими умовами за припущення, що вони підпорядковані деяким квадратичним обмеженням. За непрямими зашумленими спостереженнями їх розв'язків на скінченній системі точок та інтервалів отримано лінійні гарантовані середньоквадратичні оцінки лінійних функціоналів від невідомих даних цих задач. Встановлено, що якщо невідомі кореляційні функції похибок у спостереженнях належать деяким спеціальним множинам, то такі оцінки виражаються через розв'язки деяких крайових задач для лінійних систем імпульсних звичайних диференціальнх рівнянь.
\end{abstract}

Ключові слова: гарантована середнъоквадратична оцінка, зашумлені спостереження, лінійні функціонали від невідомих даних.

2000 MSC: $34 \mathrm{H} 05,34 \mathrm{G} 25$

УДК: 519.8 